\title{
Multiantenna spectrum sensing for Cognitive Radio: overcoming noise uncertainty
}

\author{
Roberto López-Valcarce and Gonzalo Vazquez-Vilar \\ Dept. Signal Theory \& Communications \\ University of Vigo \\ 36310 Vigo, Spain \\ email: $\{$ valcarce, gvazquez $\}$ gts.tsc.uvigo.es
}

\author{
Josep Sala \\ Dept. Signal Theory \& Communications \\ Technical University of Catalonia \\ 08034 Barcelona, Spain \\ email: josep.sala@upc.edu
}

\begin{abstract}
Spectrum sensing is a key ingredient of the dynamic spectrum access paradigm, but it needs powerful detectors operating at SNRs well below the decodability levels of primary signals. Noise uncertainty poses a significant challenge to the development of such schemes, requiring some degree of diversity (spatial, temporal, or in distribution) for identifiability of the noise level. Multiantenna detectors exploit spatial independence of receiver thermal noise. We review this class of schemes and propose a novel detector trading off performance and complexity. However, most of these methods assume that the noise power, though unknown, is the same at all antennas. As it turns out, calibration errors have a substantial impact on these detectors. Another novel detector is proposed, based on an approximation to the Generalized Likelihood Ratio, outperforming previous schemes for uncalibrated multiantenna receivers.
\end{abstract}

\section{INTRODUCTION}

The apparent scarcity of spectral resources while most allocated frequency bands are underutilized motivates the Cognitive Radio (CR) paradigm [1]. The key idea of opportunistically accessing momentarily unused licensed bands requires powerful spectrum monitoring techniques, since the interference produced to licensed (primary) users must be kept at sufficiently low levels. At the same time, the wireless medium makes reliable detection of primary users a very challenging task: due to fading and shadowing phenomena, the received primary signal may be very weak, resulting in very low Signal-to-Noise Ratio (SNR) operation conditions [2].

Certain properties of the primary signal, such as the presence of any pilots or cyclostationary features, could in principle be exploited in order to obtain powerful detectors. However, such approaches become very sensitive to synchronization errors [2]. With very low SNR, the synchronization loops of the monitoring system cannot be expected to provide the required accuracy for the carrier frequency and/or clock rate estimates. At the other end of the range of detection techniques one finds the popular Energy Detector, which has very low complexity, operates asynchronously, and does not require any a priori knowledge of primary signals. These desirable traits come at the cost of a reduced detection

This work was supported by the Spanish Government under projects SPROACTIVE (reference TEC2007-68094-C02/TCM) and COMONSENS (CONSOLIDER-INGENIO 2010 CSD2008-00010). performance [2]. However, knowledge of the noise variance is required so that the threshold to which the received signal power is compared can be computed in order to set the desired probability of false alarm. A critical SNR level (an "SNR wall" [3]) appears if the actual value of the noise variance is different from the nominal value. Primary signals below this critical value become undetectable, even if the observation time goes to infinity. This serious drawback motivates the search for asynchronous detectors robust to noise uncertainty.

In this regard, exploiting the availability of multiple antennas constitutes a promising approach. Multiple-input multipleoutput (MIMO) technologies having reached considerable maturity, it is very likely for future CR terminals to incorporate them [4], [5]. In terms of transmission/reception, multiple antennas provide a means to increase channel capacity without bandwidth expansion, as well as to overcome the effects of fading via space-time coding [6]. Several authors have recently studied the benefits of having multiple antennas in terms of enhancing detection performance in the context of CR systems. In [7] an analysis was given for multiantenna Energy Detectors using maximal ratio combining and antenna selection; these schemes require knowledge of the channel from the primary user to the cognitive node and therefore are difficult to implement in practice. A priori knowledge about the spectral characteristics of the transmitted primary signal was exploited in [8] together with a multiantenna sensor in order to develop a generalized Energy Detector under a weak signal assumption. Both [7] and [8] assume knowledge of the noise variance, and therefore they inherit the sensitivity of the original Energy Detector to uncertainties about this parameter.

However, with multiantenna sensors it is possible in principle to overcome this problem. The basic idea is to exploit the fact that, if the channel under scrutiny is being used by the primary network, then some spatial correlation should be present in the signals at different antennas. On the other hand, if the channel is idle so that the only contribution in the observations corresponds to thermal noise, then such correlation should be absent. Thus, detectors can be designed based on spatial correlation estimates, rather than received signal power. An alternative interpretation is that multiantenna sensors provide additional degrees of freedom which allow for simultaneous estimation of signal and noise parameters. This 
is not possible with single-antenna systems, unless some additional assumptions are adopted to allow distinction between signal and noise, e.g. Gaussian noise vs. non-Gaussian signal, or temporally white noise vs. correlated signal.

Several authors have considered the multiantenna detection problem under unknown noise [9]-[12] to devise schemes that remain robust to noise uncertainties. All of these detectors assume the same noise variance across the antennas. In practice, however, tolerances in the components of the different RF chains will result in deviations of the noise level from antenna to antenna. As it turns out, the detectors from [9][12] are sensitive to these calibration errors. Hence, it is of interest to devise detectors handling unknown noise variances which are potentially different at each antenna; see e.g. [13].

The contribution of this paper is twofold. First, a new multiantenna detector under unknown noise is proposed which, though suboptimal, does not require obtaining the eigenvalues of the spatial correlation matrix, in contrast to previous approaches. The performance of this detector compares favorably to eigenvalue-based detectors, but it also degrades when the noise variance is not uniform across the antennas. A second multiantenna detector is proposed for this more challenging scenario, based on an approximation to the Generalized Likelihood Ratio. This scheme performs better that the detector from [13] when the number of antennas is larger than two.

The problem setting is given in Section II. Section III presents different detectors derived assuming uniform noise variance across all antennas. Detectors designed for uncalibrated receivers are presented in Section IV. Numerical results and conclusions are given in Sections V and VI, respectively.

\section{SYSTEM MODEL}

The sensor has $M$ antennas with their respective RF chains. The same primary channel is selected at all antennas, downconverted to baseband, and asynchronously sampled. The $K$ complex-valued samples $y_{k}^{(m)}$ at the $m$ th antenna are given by

$$
y_{k}^{(m)}=h_{m}^{*} x_{k}+\sigma_{m} w_{k}^{(m)}, \quad 1 \leq m \leq M, 1 \leq k \leq K,
$$

where $x_{k}$ are the (noiseless) samples of the zero-mean primary signal, normalized to unit variance $\left(E\left\{\left|x_{k}\right|^{2}\right\}=1\right) ; w_{k}^{(m)}$ are samples of a zero mean, circular complex white Gaussian noise also with unit variance; $h_{m}^{*}$ and $\sigma_{m}^{2}>0$ are respectively the complex channel gain and noise power at antenna $m$ (if the channel is vacant, then $h_{m}^{*}=0$ for all $m$ ). The noise processes at different antennas are assumed independent. Let $\boldsymbol{Y} \in \mathbb{C}^{K \times M}$ be the data matrix whose $(k, m)$-th element is $y_{k}^{(m)}$. Then we can rewrite (1) as

$$
\boldsymbol{Y}=\boldsymbol{x} \boldsymbol{h}^{H}+\boldsymbol{W} \boldsymbol{\Sigma},
$$

where the $(k, m)$-th element of $\boldsymbol{W} \in \mathbb{C}^{K \times M}$ is $w_{k}^{(m)}$, and

$$
\boldsymbol{x} \doteq\left[\begin{array}{c}
x_{1} \\
x_{2} \\
\vdots \\
x_{K}
\end{array}\right], \boldsymbol{h} \doteq\left[\begin{array}{c}
h_{1} \\
\vdots \\
h_{M}
\end{array}\right], \boldsymbol{\Sigma} \doteq\left[\begin{array}{ccc}
\sigma_{1} & & \\
& \ddots & \\
& & \sigma_{M}
\end{array}\right]
$$

Note that the model above assumes that the channel from the primary transmitter to the spectrum monitor is frequency flat in the RF channel bandwidth, and that it remains constant for the duration of the sensing time. In addition, for the sake of tractability the following assumptions are adopted:

- A1: The primary signal follows a Gaussian distribution.

- A2: The primary signal is temporally white.

If the modulation format of the primary system is Orthogonal Frequency Division Multiplexing (OFDM), then Assumption A1 approximately holds for the number of subcarriers used in practice. Noting that the spectrum of an OFDM signal is approximately constant within the signal bandwidth, Assumption A2 will be approximately satisfied if the sampling frequency of the system is set to twice the baseband bandwidth of the primary transmission (i.e. equal to the RF channel bandwidth minus the guard bands).

If primary users employ single-carrier modulation and pulse shaping with excess bandwidth, the assumptions above will not strictly hold. In that case, exploiting time correlation and/or non-Gaussianity of the primary signal could result in more powerful detection schemes. Nevertheless, the Gaussian model still provides practical detectors for this scenario.

Under Assumptions A1 and A2, the observation processes $y_{k}^{(m)}, 1 \leq m \leq M$, are temporally white and Gaussian, and the only dependence of the probability density function (pdf) of $\boldsymbol{Y}$ is through the sample correlation matrix

$$
\hat{\boldsymbol{R}} \doteq \frac{1}{K} \boldsymbol{Y}^{H} \boldsymbol{Y}
$$

Then, with $\boldsymbol{R} \doteq E\{\hat{\boldsymbol{R}}\}=\boldsymbol{\Sigma}^{2}+\boldsymbol{h} \boldsymbol{h}^{H}$, this pdf is given by

$$
f(\boldsymbol{Y} \mid \boldsymbol{R})=\left[\frac{1}{\pi \operatorname{det} \boldsymbol{R}} \exp \left\{-\operatorname{tr}\left(\boldsymbol{R}^{-1} \hat{\boldsymbol{R}}\right)\right\}\right]^{K} \text {. }
$$

\section{Calibrated multiantenna Detectors}

The schemes presented in this section assume that the noise variance is the same at all of the $M$ antennas, i.e. $\boldsymbol{\Sigma}=\sigma \boldsymbol{I}$.

\section{A. Mean/max eigenvalue test}

The corresponding hypothesis test using model (2) is

$$
\mathcal{H}_{0}: \boldsymbol{R}=\boldsymbol{R}_{0} \doteq \sigma^{2} \boldsymbol{I}, \quad \mathcal{H}_{1}: \boldsymbol{R}=\boldsymbol{R}_{1} \doteq \sigma^{2} \boldsymbol{I}+\boldsymbol{h} \boldsymbol{h}^{H}
$$

Since the parameters $\sigma^{2}, \boldsymbol{h}$ are unknown, a sensible approach is the Generalized Likelihood Ratio Test (GLRT). In the GLRT, the unknown parameters are substituted by their Maximum Likelihood (ML) estimates under each hypothesis:

$$
\ell \doteq-2 \log \frac{\max _{\boldsymbol{R}_{0}} f\left(\boldsymbol{Y} \mid \boldsymbol{R}_{0}\right)}{\max _{\boldsymbol{R}_{1}} f\left(\boldsymbol{Y} \mid \boldsymbol{R}_{1}\right)} \underset{\mathcal{H}_{0}}{\stackrel{\mathcal{H}_{1}}{\gtrless}} \gamma,
$$

where $\gamma$ is a suitable threshold. The GLRT for this model has been proposed in [12], and we briefly review it now. Under $\mathcal{H}_{0}$, the ML estimate of $\sigma^{2}$ is

$$
\hat{\sigma}_{0}^{2}=\frac{1}{M} \operatorname{tr} \hat{\boldsymbol{R}}
$$


Under $\mathcal{H}_{1}$, the ML estimates of $\sigma^{2}$ and $\boldsymbol{h}$ are given by

$$
\hat{\sigma}_{1}^{2}=\frac{\operatorname{tr} \hat{\boldsymbol{R}}-\lambda_{1}(\hat{\boldsymbol{R}})}{M-1}, \quad \hat{\boldsymbol{h}}_{1}=\sqrt{\lambda_{1}(\hat{\boldsymbol{R}})-\hat{\sigma}_{1}^{2}} \cdot \boldsymbol{v}
$$

where $\lambda_{1}(\hat{\boldsymbol{R}})$ is the largest eigenvalue of $\hat{\boldsymbol{R}}$ and $\boldsymbol{v}$ is the corresponding unit-norm eigenvector. Note that $\hat{\sigma}_{0}^{2}$ is the mean of the $M$ eigenvalues of $\hat{\boldsymbol{R}}$, whereas $\hat{\sigma}_{1}^{2}$ is the mean of the $M-1$ smallest eigenvalues of $\hat{\boldsymbol{R}}$. Substituting (8)-(9) back in (7), one obtains

$\ell=2 K \log \frac{(M-1)^{M-1} \mu^{M}}{(M \mu-1)^{M-1}}$, with $\mu \doteq \frac{\frac{1}{M} \operatorname{tr} \hat{\boldsymbol{R}}}{\lambda_{1}(\hat{\boldsymbol{R}})} \in\left[\frac{1}{M}, 1\right]$

Noting that $\ell$ is a monotonically decreasing function of $\mu$, the GLRT can be equivalently expressed as $\mu \gtrless \mathcal{H}_{\mathcal{H}_{0}} \gamma^{\prime}$. We refer to this scheme as "Mean/Max test", since the statistic $\mu$ is the ratio of the mean of the eigenvalues of $\hat{\boldsymbol{R}}$ to the largest one.

\section{B. Sphericity test}

If no structure is assumed about the covariance matrix when the signal is present, the following test results:

$$
\mathcal{H}_{0}: \boldsymbol{R}=\boldsymbol{R}_{0} \doteq \sigma^{2} \boldsymbol{I}, \quad \mathcal{H}_{1}: \boldsymbol{R}=\boldsymbol{R}_{1}=\boldsymbol{R}_{1}^{H}>\mathbf{0} .
$$

The ML estimate of $\sigma^{2}$ under $\mathcal{H}_{0}$ is given by (8), whereas the ML estimate of $\boldsymbol{R}$ under $\mathcal{H}_{1}$ is $\hat{\boldsymbol{R}}_{1}=\hat{\boldsymbol{R}}$. This results in the following GLRT:

$$
\ell=2 K M \log \frac{\frac{1}{M} \operatorname{tr} \hat{\boldsymbol{R}}}{\sqrt[M]{\operatorname{det} \hat{\boldsymbol{R}}}},
$$

which is known as the "test for sphericity" [10], [14]. Note that the statistic for the GLRT in this case reduces to the ratio of the arithmetic to geometric mean of the eigenvalues of $\hat{\boldsymbol{R}}$.

\section{Ad hoc eigenvalue tests}

Both the Mean/Max and Sphericity tests are based on metrics on the eigenvalues of $\hat{\boldsymbol{R}}$ that measure the distance from this matrix to a scaled identity, for which all eigenvalues are equal. Two other tests have been proposed in [11] based on alternative metrics of this kind: the "dispersion test" (i.e. ratio of smallest to largest eigenvalues $\lambda_{M} / \lambda_{1}$ ), and the "Min/Mean test" $\lambda_{M} /\left(\frac{1}{M} \operatorname{tr} \hat{\boldsymbol{R}}\right)$. Here we propose another detector based on the following property of the trace operator:

Property 1: For any Hermitian $\boldsymbol{A} \in \mathbb{C}^{M \times M}$, it holds that

$$
0 \leq \frac{\operatorname{tr}^{2}(\boldsymbol{A})}{M \operatorname{tr}\left(\boldsymbol{A}^{H} \boldsymbol{A}\right)} \leq 1
$$

and the upper bound is attained iff $\boldsymbol{A}=\alpha \boldsymbol{I}$.

The proof follows by invoking the Cauchy-Schwarz inequality for the inner product $\langle\boldsymbol{A}, \boldsymbol{B}\rangle \doteq \operatorname{tr}\left(\boldsymbol{B}^{H} \boldsymbol{A}\right)$ with $\boldsymbol{B}=\boldsymbol{I}$.

Hence, the following "Trace/Trace" test can be applied to this detection problem:

$$
\frac{\operatorname{tr}^{2}(\hat{\boldsymbol{R}})}{M \operatorname{tr}\left(\hat{\boldsymbol{R}}^{H} \hat{\boldsymbol{R}}\right)} \stackrel{\mathcal{H}_{1}}{\gtrless} \gamma .
$$

Note that (14) does not require eigenvalue extraction.

\section{Two-antenna sensors}

When $M=2$, the following relations are readily checked:

$$
\begin{array}{rlrl}
\frac{\operatorname{tr} \hat{\boldsymbol{R}}}{M \lambda_{1}} & =\frac{1+\nu}{2}, & & \frac{\frac{1}{M} \operatorname{tr} \hat{\boldsymbol{R}}}{M}=\frac{1+\nu}{2 \sqrt{\operatorname{det} \hat{\boldsymbol{R}}}}, \\
\frac{\lambda_{M}}{\frac{1}{M} \operatorname{tr} \hat{\boldsymbol{R}}}=\frac{2 \nu}{1+\nu}, & \frac{\operatorname{tr}^{2}(\hat{\boldsymbol{R}})}{M \operatorname{tr}\left(\hat{\boldsymbol{R}}^{2}\right)}=\frac{(1+\nu)^{2}}{2\left[1+\nu^{2}\right]},
\end{array}
$$

which are all monotonic functions of the dispersion $\nu \doteq$ $\lambda_{2} / \lambda_{1} \in[0,1]$. Therefore, for the important case of a sensor with two antennas, the Mean/Max, Sphericity, Min/Mean, Dispersion, and Trace/Trace tests are all equivalent.

\section{DETECTORS FOR UNCALIBRATED RECEIVERS}

Now we relax the assumption of uniform noise variances across the antennas, so that $\boldsymbol{\Sigma}$ is any positive diagonal matrix.

\section{A. Hadamard ratio test}

The following test makes no assumption on the structure of the covariance matrix when the signal is present:

$$
\mathcal{H}_{0}: \boldsymbol{R}=\boldsymbol{R}_{0} \doteq \boldsymbol{\Sigma}^{2}, \quad \mathcal{H}_{1}: \boldsymbol{R}=\boldsymbol{R}_{1}=\boldsymbol{R}_{1}^{H}>\mathbf{0} .
$$

The ML estimate of $\boldsymbol{\Sigma}^{2}$ under $\mathcal{H}_{0}$ is $\hat{\boldsymbol{\Sigma}}_{0}^{2}=\operatorname{diag} \hat{\boldsymbol{R}}$, whereas the ML estimate of $\boldsymbol{R}$ under $\mathcal{H}_{1}$ is $\hat{\boldsymbol{R}}_{1}=\hat{\boldsymbol{R}}$. This yields the following GLRT [13]:

$$
\ell=-2 K M \log \frac{\operatorname{det} \hat{\boldsymbol{R}}}{\operatorname{det} \operatorname{diag} \hat{\boldsymbol{R}}}=-2 K M \log \operatorname{det} \hat{\boldsymbol{C}},
$$

where we have introduced the sample correlation matrix (also known as spatial coherence matrix)

$$
\hat{\boldsymbol{C}} \doteq[\operatorname{diag} \hat{\boldsymbol{R}}]^{-1 / 2} \hat{\boldsymbol{R}}[\operatorname{diag} \hat{\boldsymbol{R}}]^{-1 / 2} .
$$

Note that $\hat{\boldsymbol{C}}$ has ones on the diagonal, and that its $(m, n)$ th element is just the sample correlation coefficient between antennas $m$ and $n$. Note that the (squared) Frobenius norm $\|\hat{\boldsymbol{C}}\|_{F}^{2}$ is therefore (twice) the sum of the squares of the correlation coefficients of all antenna pairs, plus a constant term, and constitutes a plausible statistic for this hypothesis test. In fact, it was shown in [13] that, for weak signals, the Frobenius norm test and the "Hadamard ratio" test based on (18) are equivalent in terms of detection performance; computational complexity favors the Frobenius norm test. Finally, note that the approach from Section III-C could be applied here in order to derive a detector that measures the distance between $\hat{C}$ and the identity: the resulting scheme turns out to be equivalent to the Frobenius norm test, since $\operatorname{tr}^{2}(\hat{\boldsymbol{C}})=M^{2}$ and $\operatorname{tr}\left(\hat{\boldsymbol{C}}^{H} \hat{\boldsymbol{C}}\right)=\|\hat{\boldsymbol{C}}\|_{F}^{2}$.

\section{B. A new detector}

The corresponding hypothesis test using model (2) is

$\mathcal{H}_{0}: \boldsymbol{R}=\boldsymbol{R}_{0} \doteq \boldsymbol{\Sigma}^{2}, \quad \mathcal{H}_{1}: \boldsymbol{R}=\boldsymbol{R}_{1} \doteq \boldsymbol{\Sigma}^{2}+\boldsymbol{h} \boldsymbol{h}^{H}$.

Thus, under $\mathcal{H}_{1}$, the covariance matrix is "diagonal plus rank one". Again, the ML estimate of $\Sigma^{2}$ under $\mathcal{H}_{0}$ is $\hat{\boldsymbol{\Sigma}}_{0}^{2}=\operatorname{diag} \hat{\boldsymbol{R}}$, yielding

$$
\log \operatorname{det} \boldsymbol{R}_{0}+\operatorname{tr}\left(\boldsymbol{R}_{0}^{-1} \hat{\boldsymbol{R}}\right)=M+\log \operatorname{det} \operatorname{diag} \hat{\boldsymbol{R}} .
$$


In order to obtain the ML estimates of $\boldsymbol{\Sigma}^{2}$ and $\boldsymbol{h}$ under $\mathcal{H}_{1}$, note that the following two facts hold:

$$
\begin{aligned}
\operatorname{det}\left(\boldsymbol{\Sigma}^{2}+\boldsymbol{h} \boldsymbol{h}^{H}\right) & =\left(1+\boldsymbol{h}^{H} \boldsymbol{\Sigma}^{-2} \boldsymbol{h}\right) \operatorname{det} \boldsymbol{\Sigma}^{2}, \\
\left(\boldsymbol{\Sigma}^{2}+\boldsymbol{h} \boldsymbol{h}^{H}\right)^{-1} & =\boldsymbol{\Sigma}^{-2}-\frac{\boldsymbol{\Sigma}^{-2} \boldsymbol{h} \boldsymbol{h}^{H} \boldsymbol{\Sigma}^{-2}}{1+\boldsymbol{h}^{H} \boldsymbol{\Sigma}^{-2} \boldsymbol{h}} .
\end{aligned}
$$

Hence, the negative of the log-likelihood function, which is to be minimized under $\mathcal{H}_{1}$ w.r.t. $\boldsymbol{\Sigma}^{2}$ and $\boldsymbol{h}$, is given by

$$
\begin{aligned}
\log \operatorname{det} \boldsymbol{R}_{1} & +\operatorname{tr}\left(\boldsymbol{R}_{1}^{-1} \hat{\boldsymbol{R}}\right)=\log \operatorname{det} \boldsymbol{\Sigma}^{2}+\operatorname{tr}\left(\boldsymbol{\Sigma}^{-2} \hat{\boldsymbol{R}}\right) \\
& +\log \left(1+\boldsymbol{h}^{H} \boldsymbol{\Sigma}^{-2} \boldsymbol{h}\right)-\frac{\boldsymbol{h}^{H} \boldsymbol{\Sigma}^{-2} \hat{\boldsymbol{R}} \boldsymbol{\Sigma}^{-2} \boldsymbol{h}}{1+\boldsymbol{h}^{H} \boldsymbol{\Sigma}^{-2} \boldsymbol{h}} .
\end{aligned}
$$

Let us define now

$$
\boldsymbol{g} \doteq \frac{\boldsymbol{\Sigma}^{-2} \boldsymbol{h}}{\sqrt{\boldsymbol{h}^{H} \boldsymbol{\Sigma}^{-2} \boldsymbol{h}}}, \quad \alpha^{2} \doteq \boldsymbol{h}^{H} \boldsymbol{\Sigma}^{-2} \boldsymbol{h},
$$

and note that $\boldsymbol{g}^{H} \boldsymbol{\Sigma}^{2} \boldsymbol{g}=1$. Then (24) reads as

$$
\begin{array}{r}
\log \operatorname{det} \boldsymbol{R}_{1}+\operatorname{tr}\left(\boldsymbol{R}_{1}^{-1} \hat{\boldsymbol{R}}\right)=\log \operatorname{det} \boldsymbol{\Sigma}^{2}+\operatorname{tr}\left(\boldsymbol{\Sigma}^{-2} \hat{\boldsymbol{R}}\right) \\
+\log \left(1+\alpha^{2}\right)-\frac{\alpha^{2}}{1+\alpha^{2}} \boldsymbol{g}^{H} \hat{\boldsymbol{R}} \boldsymbol{g},
\end{array}
$$

which is minimized w.r.t. $\boldsymbol{g}$ (subject to $\boldsymbol{g}^{H} \boldsymbol{\Sigma}^{2} \boldsymbol{g}=1$ ) when $\boldsymbol{g}$ is the eigenvector of $\boldsymbol{\Sigma}^{-2} \hat{\boldsymbol{R}}$ associated to its largest eigenvalue and satisfying the constraint. This choice yields $\boldsymbol{g}^{H} \hat{\boldsymbol{R}} \boldsymbol{g}=$ $\lambda_{1}\left(\boldsymbol{\Sigma}^{-2} \hat{\boldsymbol{R}}\right)$ and

$$
\begin{array}{r}
\log \operatorname{det} \boldsymbol{R}_{1}+\operatorname{tr}\left(\boldsymbol{R}_{1}^{-1} \hat{\boldsymbol{R}}\right)=\log \operatorname{det} \boldsymbol{\Sigma}^{2}+\operatorname{tr}\left(\boldsymbol{\Sigma}^{-2} \hat{\boldsymbol{R}}\right) \\
+\log \left(1+\alpha^{2}\right)-\frac{\alpha^{2}}{1+\alpha^{2}} \lambda_{1}\left(\boldsymbol{\Sigma}^{-2} \hat{\boldsymbol{R}}\right)
\end{array}
$$

which in turn is minimized w.r.t. $\alpha^{2}$ for $\alpha^{2}=\lambda_{1}\left(\boldsymbol{\Sigma}^{-2} \hat{\boldsymbol{R}}\right)-1$. Substituting this in (27), the expression obtained depends only on $\boldsymbol{\Sigma}^{2}$ and can be written as

$\log \operatorname{det} \boldsymbol{R}_{1}+\operatorname{tr}\left(\boldsymbol{R}_{1}^{-1} \hat{\boldsymbol{R}}\right)=1+\log \operatorname{det} \hat{\boldsymbol{R}}-(M-1)$

$\times\left[\log \left(\frac{\operatorname{det}\left(\boldsymbol{\Sigma}^{-2} \hat{\boldsymbol{R}}\right)}{\lambda_{1}\left(\boldsymbol{\Sigma}^{-2} \hat{\boldsymbol{R}}\right)}\right)^{\frac{1}{M-1}}-\frac{\operatorname{tr}\left(\boldsymbol{\Sigma}^{-2} \hat{\boldsymbol{R}}\right)-\lambda_{1}\left(\boldsymbol{\Sigma}^{-2} \hat{\boldsymbol{R}}\right)}{M-1}\right]$.

Now the bracketed term in (28) must be maximized w.r.t. $\boldsymbol{\Sigma}^{2}$ (diagonal and positive definite). Note that this term can be written in terms of the arithmetic and geometric means of the $M-1$ smallest eigenvalues of $\boldsymbol{\Sigma}^{-2} \hat{\boldsymbol{R}}$ as

$$
\begin{aligned}
\log \left[\prod_{i=2}^{M} \lambda_{i}\left(\boldsymbol{\Sigma}^{-2} \hat{\boldsymbol{R}}\right)\right]^{\frac{1}{M-1}}-\frac{\sum_{j=2}^{M} \lambda_{j}\left(\boldsymbol{\Sigma}^{-2} \hat{\boldsymbol{R}}\right)}{M-1} \\
=-\frac{1}{M-1} \sum_{i=2}^{M}\left[\lambda_{i}\left(\boldsymbol{\Sigma}^{-2} \hat{\boldsymbol{R}}\right)-\log \lambda_{i}\left(\boldsymbol{\Sigma}^{-2} \hat{\boldsymbol{R}}\right)\right] .
\end{aligned}
$$

There is no closed-form general solution for the diagonal $\boldsymbol{\Sigma}^{2}$ maximizing (29). Note, however, that the maximum of (29) w.r.t. $\lambda_{2}, \ldots, \lambda_{M}$ is attained when $\lambda_{2}=\cdots=\lambda_{M}=1$. But this point is not reachable in general, since the eigenvalues of $\boldsymbol{\Sigma}^{-2} \hat{\boldsymbol{R}}$ cannot be arbitrarily selected by choice of $\boldsymbol{\Sigma}^{2}$. An exception occurs if $\hat{\boldsymbol{R}}$ is diagonal: in that case, the maximum is attained when $\boldsymbol{\Sigma}^{2}=\operatorname{diag} \hat{\boldsymbol{R}}$. Under a weak signal assumption the matrix $\hat{\boldsymbol{R}}$ will be close to diagonal, and therefore $\boldsymbol{\Sigma}^{2}=$ $\operatorname{diag} \hat{\boldsymbol{R}}$ will be close to optimal. With this choice, and noting that the eigenvalues of $[\operatorname{diag} \hat{\boldsymbol{R}}]^{-2} \hat{\boldsymbol{R}}$ are the same as those of $\hat{C}$ from (19), the bracketed term in (28) reduces to

$$
\frac{1}{M-1} \log \frac{\operatorname{det}(\hat{\boldsymbol{C}})}{\lambda_{1}(\hat{\boldsymbol{C}})}-\frac{M-\lambda_{1}(\hat{\boldsymbol{C}})}{M-1},
$$

and (28) becomes

$$
\begin{aligned}
\log \operatorname{det} \boldsymbol{R}_{1}+\operatorname{tr}\left(\boldsymbol{R}_{1}^{-1} \hat{\boldsymbol{R}}\right)= & M+1+\log \frac{\operatorname{det} \hat{\boldsymbol{R}}}{\operatorname{det} \hat{\boldsymbol{C}}} \\
& -\lambda_{1}(\hat{\boldsymbol{C}})+\log \lambda_{1}(\hat{\boldsymbol{C}})
\end{aligned}
$$

From (21) and (31), and after taking into account that $(\operatorname{det} \operatorname{diag} \hat{\boldsymbol{R}})(\operatorname{det} \hat{\boldsymbol{C}})=\operatorname{det}[(\operatorname{diag} \hat{\boldsymbol{R}}) \hat{\boldsymbol{C}}]=\operatorname{det} \hat{\boldsymbol{R}}$, the resulting approximate GLRT that results is given by

$$
\begin{aligned}
\ell & =2 K\left[\log \frac{\operatorname{det} \boldsymbol{R}_{0}}{\operatorname{det} \boldsymbol{R}_{1}}+\operatorname{tr}\left(\boldsymbol{R}_{0}^{-1}-\boldsymbol{R}_{1}^{-1}\right) \hat{\boldsymbol{R}}\right] \\
& \approx 2 K\left[-1+\lambda_{1}(\hat{\boldsymbol{C}})-\log \lambda_{1}(\hat{\boldsymbol{C}})\right] .
\end{aligned}
$$

Since $\lambda_{1}(\hat{\boldsymbol{C}}) \geq \frac{1}{M} \operatorname{tr} \hat{\boldsymbol{C}}=1$, and $f(x)=-1+x-\log x$ is monotonically increasing in $x \geq 1$, the proposed test is equivalently given by $\lambda_{1}(\hat{\boldsymbol{C}}) \gtrless \mathcal{H}_{\mathcal{H}_{0}} \gamma^{\prime}$.

\section{Two-antenna sensors}

The statistics of the detectors of Sections IV-A and IV-B are functions of the sample correlation matrix $\hat{C}$ only. When $M=2$, this matrix is given by $\hat{\boldsymbol{C}}=\left[\begin{array}{cc}1 & \hat{\rho} \\ \hat{\rho}^{*} & 1\end{array}\right]$, where $\hat{\rho}$ is the sampled correlation coefficient between the two antennas. Since $\operatorname{det} \hat{\boldsymbol{C}}=1-|\hat{\rho}|^{2}$ and $\lambda_{1}(\hat{\boldsymbol{C}})=1+|\hat{\rho}|$, which are both functions of $|\hat{\rho}|$, it follows that for two-antenna arrays both detectors are equivalent.

\section{NUMERICAL RESULTS}

\section{A. Two-antenna sensors}

The Receiver Operation Characteristic (ROC) curves obtained for the different detectors with $M=2$ are shown in Fig. 1. The SNR at the $m$-th antenna is defined as $\mathrm{SNR}_{m} \doteq\left|h_{m}\right|^{2} / \sigma_{m}^{2}$. Without noise power mismatch (i.e. same noise variance at both antennas), the detectors of Section III (which, as expected, are all equivalent) outperform those of Section IV. This is reasonable, since in these conditions the schemes from Section III exploit more efficiently the data model. However, with $\sigma_{2}^{2}=1.15 \sigma_{1}^{2}$ (0.6 dB mismatch), the performance of these detectors degrades substantially, whereas that of the methods of Section IV changes only slightly. Fig. 2 shows the variation in probability of false alarm $P_{\mathrm{FA}}$ for the Mean/Max detector under noise mismatch; together with Fig. 1, this shows that, once a threshold is set, the performance of this scheme (and the equivalent ones) degrades in terms of both $P_{\mathrm{FA}}$ and probability of detection $P_{\mathrm{D}}$. On the other hand, the tests of Section IV maintain the same $P_{\mathrm{FA}}$ for a given threshold under noise mismatch conditions. 


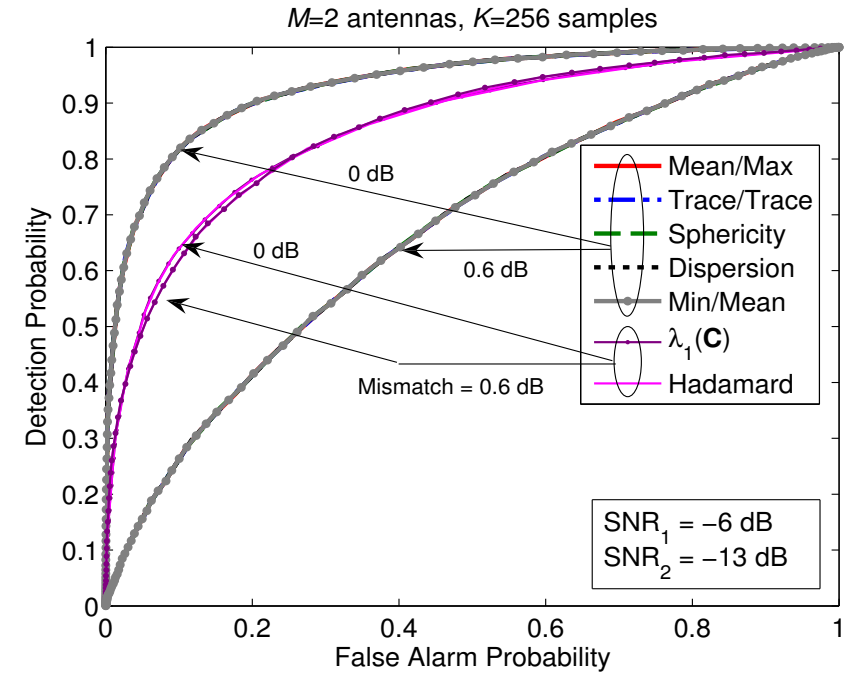

Fig. 1. ROC for the different detectors $(M=2)$ with and without noise power mismatch.

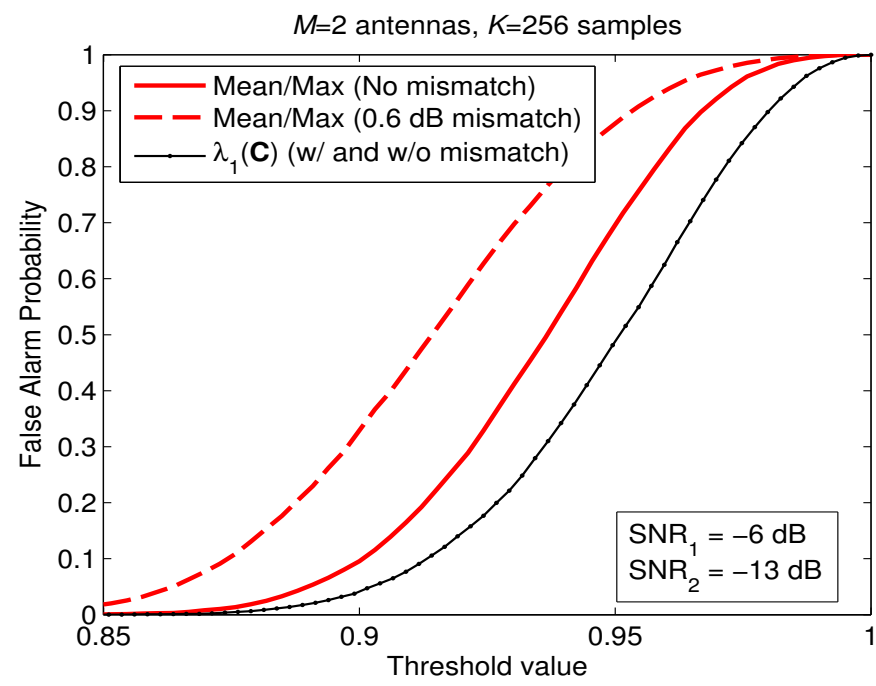

Fig. 2. $\quad P_{\mathrm{FA}}$ for the different detectors $(M=2)$ with and without noise power mismatch.

\section{B. Sensors with more than two antennas}

When $M>2$ the different detectors are not equivalent in general. The corresponding ROC curves are shown in Fig. 3 for a uniform noise variance scenario. The Mean/Max test, corresponding to the GLRT under this model, yields the best performance, followed by the Trace/Trace detector (14). Among the ad hoc schemes, the Min/Mean detector shows the poorest performance. On the other hand, of the two detectors designed for uncalibrated receivers, it is seen that the novel scheme based on $\lambda_{1}(\hat{\boldsymbol{C}})$ outperforms the Hadamard ratio detector. This is also the case with noise mismatch, as seen in Figs. 4 and 5 for two different sets of mismatch parameters. The performance loss of the schemes from Section III is also clear. Note also that, depending on the interantenna mismatch values, the GLRT-based Mean/Max detector may

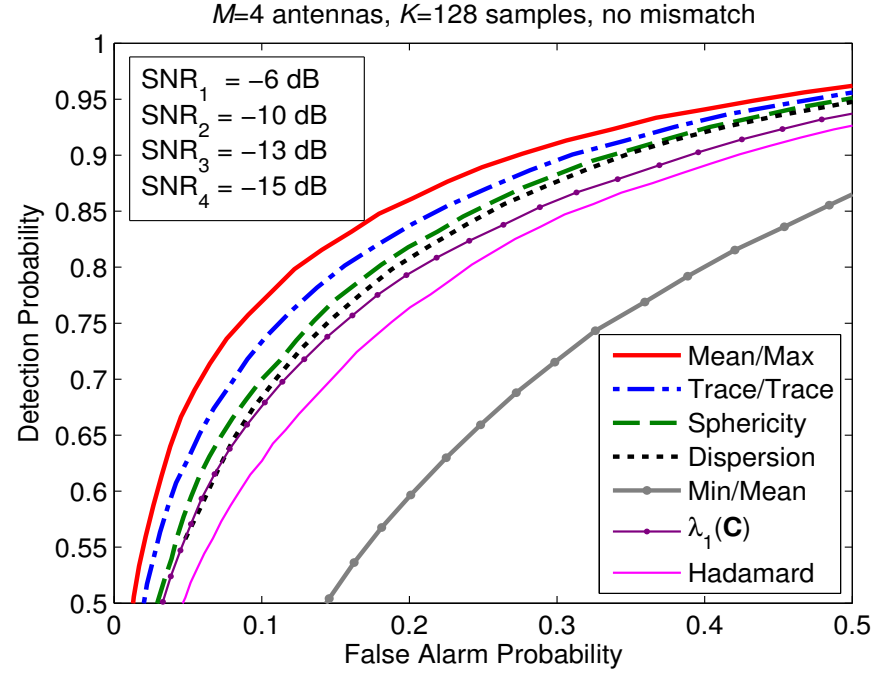

Fig. 3. ROC for the different detectors $(M=4)$ without noise power mismatch.

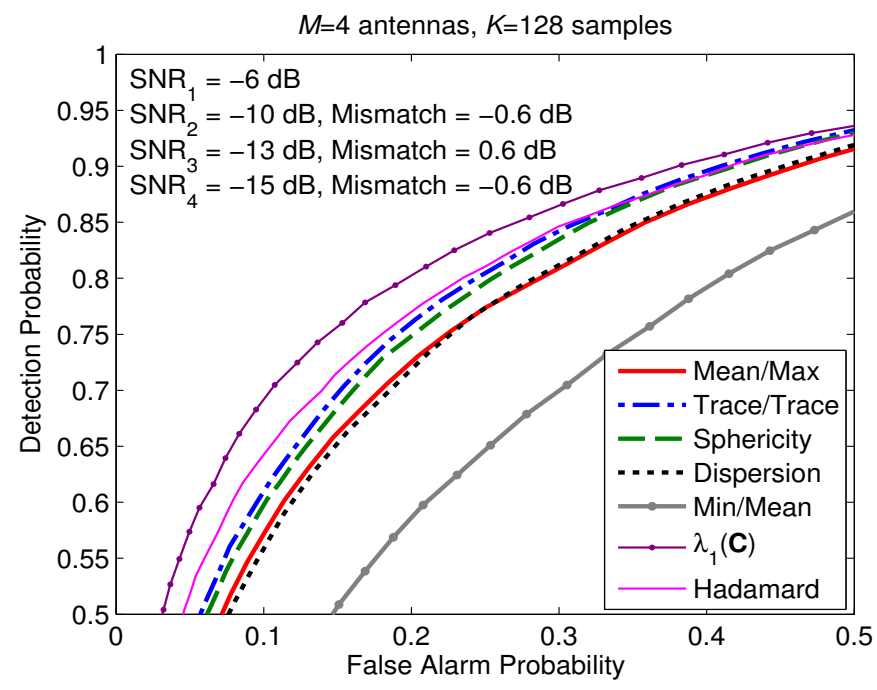

Fig. 4. ROC for the different detectors $(M=4)$ with noise power mismatch.

perform worse than some ad hoc methods (e.g. the Trace/Trace and Sphericity detectors in Fig. 4).

\section{Frequency selective channels}

To show the robustness of the different detectors against (unknown) frequency selective channels we consider the same scenario as in Fig. 5, but now under multipath conditions. The channel frequency response ${ }^{1}$ at each of the four antennas is shown in Fig. 6. Fig. 7 shows the ROC curves corresponding to the detectors $\lambda_{1}(\hat{\boldsymbol{C}})$, Hadamard and Mean/Max for both the frequency flat and frequency selective scenarios. The relative advantage of the schemes from Section IV with respect to Mean/Max remains unaltered. However, for all the

\footnotetext{
${ }^{1}$ Obtained using the WINNER Phase II Model [15]: Profile C1 (Suburban); central frequency $800 \mathrm{MHz} ; 8 \mathrm{MHz}$ bandwidth; receiver antenna elements placed in a uniform linear array (UL A) with $1 \mathrm{~cm}$ spacing.
} 


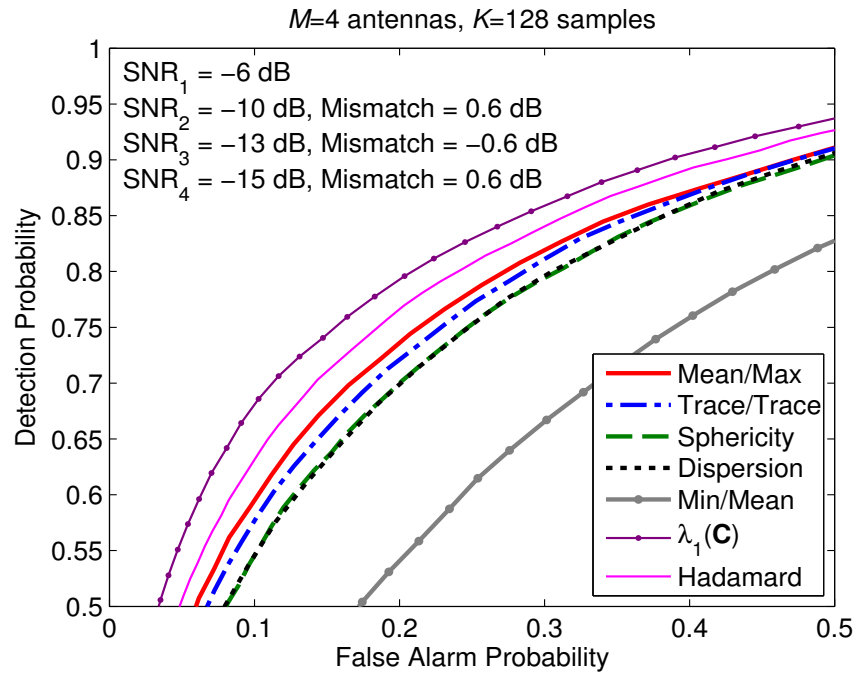

Fig. 5. ROC for the different detectors $(M=4)$ with noise power mismatch.

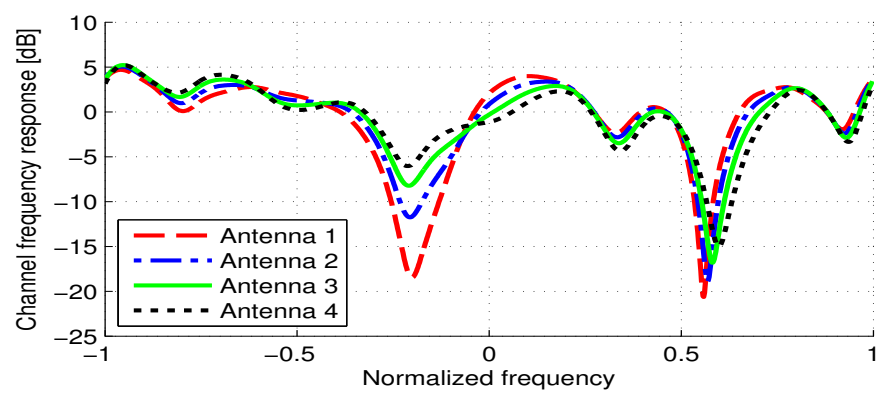

Fig. 6. Channel frequency response in a multipath scenario.

detectors considered there is a small perfomance loss in terms of probability of detection with respect to a frequency-flat scenario due to the induced temporal correlation. Note that the probability of false alarm does not change, since the statistic under hypothesis $\mathcal{H}_{0}$ depends only on the noise and not on the received signal.

\section{CONCLUSIONS}

It is a common belief that with multiple antennas, the noise uncertainty problem affecting the standard Energy Detector can be overcome. While this is true, it must be realized that certain multiantenna detectors may be sensitive to nonuniform noise variance across the antennas. We have revised several multiantenna schemes from the literature, showing that this is the case indeed.

As usually the case with detectors derived under the GLRT approach, it is difficult in general to obtain analytical expressions for the probabilities of detection and false alarm, which in most cases must be obtained by Monte Carlo simulation. As an exception, the result from [14, Th. 5.2.1] can be applied to certain cases in order to obtain the probability of false alarm of GLRT detectors: for example, the statistics (12) (sphericity test) and (18) (Hadamard ratio test) follow respectively $\chi_{M^{2}-1}^{2}$ and $\chi_{M^{2}-M}^{2}$ distributions under $\mathcal{H}_{0}$. Other approaches from

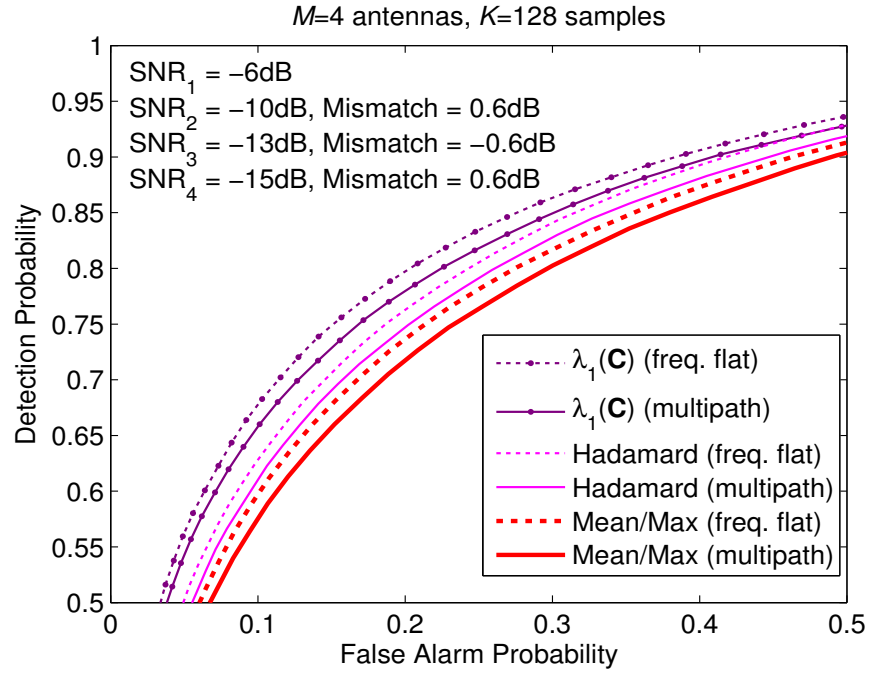

Fig. 7. ROC for the different detectors $(M=4)$ with noise power mismatch under frequency selective fading.

random matrix theory can also be useful to analytically characterize the performance of these detectors [12].

\section{REFERENCES}

[1] Q. Zhao and B. M. Sadler, "A survey of dynamic spectrum access," IEEE Signal Processing Mag., vol. 24, no. 3, pp. 79-89, May 2007.

[2] D. Cabric, "Addressing the feasibility of cognitive radios," IEEE Signal Processing Mag., vol. 25, no. 6, pp. 85-93, Nov. 2008.

[3] R. Tandra and A. Sahai, "SNR walls for signal detection," IEEE J. Sel. Top. Signal Process., vol. 2, no. 1, pp. 4-17, Feb 2008.

[4] R. Zhang and Y.-C. Liang, "Exploiting multi-antennas for opportunistic spectrum sharing in cognitive radio networks," IEEE J. Sel. Topics Signal Process., vol. 2, no. 1, pp. 88-102, Feb. 2008.

[5] G. Scutari, D. P. Palomar, and S. Barbarossa, "Cognitive MIMO radio," IEEE Signal Processing Mag., vol. 25, no. 6, pp. 46-59, Nov. 2008.

[6] E. G. Larsson and P. Stoica, Space-Time Block Coding for Wireless Communications. Cambridge University Press, 2003.

[7] A. Pandharipande and J.-P. Linnartz, "Performance analysis of primary user detection in a multiple antenna cognitive radio," Proc. IEEE Int. Conf. Communications (ICC), pp. 6482-6486, June 2007.

[8] R. López-Valcarce and G. Vazquez-Vilar, "Multiantenna detection of multicarrier primary signals exploiting spectral a priori information," Proc. 4th Int. Conf. on Cognitive Radio Oriented Wireless Networks and Communications, June 2009.

[9] Y. Zeng, Y.-C. Liang, and R. Zhang, "Blindly combined energy detection for spectrum sensing in cognitive radio," IEEE Signal Processing Lett., vol. 15 , pp. 649-652, 2008.

[10] M. Alamgir, M. Faulkner, J. Gao, and P. Conder, "Signal detection for cognitive radio using multiple antennas," Proc. IEEE Int. Symp. Wireless Comm. Systems, 2008.

[11] Y. Zeng and Y.-C. Liang, "Eigenvalue-based spectrum sensing algorithms for cognitive radio," IEEE Trans. Commun., vol. 57, no. 6, pp. 1784-1793, June 2009.

[12] A. Taherpour, M. Nasiri-Kenari, and S. Gazor, "Multiple antenna spectrum sensing in cognitive radios," IEEE Trans. Wireless Commun., 2010, accepted for publication.

[13] A. Leshem and A.-J. van der Veen, "Multichannel detection of Gaussian signals with uncalibrated receivers," IEEE Signal Processing Lett., vol. 8, no. 4, pp. 120-122, April 2001.

[14] K. V. Mardia, J. T. Kent, and J. M. Bibby, Multivariate Analysis. New York: Academic Press, 1979.

[15] L. Hentilä, P. Kyösti, M. Käske, M. Narandzic, and M. Alatossava, "MATLAB implementation of the WINNER Phase ii Channel Model ver 1.1," Online: https://www.ist-winner.org/phase_2_model.html, December 2007. 